\title{
HBME-1 expression in differentiated thyroid carcinoma and its correlation with the ultrasonic manifestation of thyroid
}

\author{
JIWEI QIAO ${ }^{1 *}$, CHUNYU LI $^{1 *}$, YUYING ZHANG $^{1}$, SHUANGYAN WANG ${ }^{2}$ and SHAN GAO ${ }^{1}$ \\ ${ }^{1}$ Longnan Hospital of Daqing City, Daqing, Heilongjiang 163453; \\ ${ }^{2}$ Daqing Hospital of Traditional Chinese Medicine, Daqing, Heilongjiang 163312, P.R. China
}

Received June 7, 2017; Accepted September 5, 2017

DOI: 10.3892/ol.2017.6971

\begin{abstract}
We investigated expression of the protein human bone marrow endothelial cell marker-1 (HBME-1) in differentiated thyroid carcinoma tissues, and analyzed its correlation with ultrasonic manifestation of thyroid. The immunohistochemistry (IHC) staining method was used to measure the expression of HBME-1 in patient with nodular goiter (control group), papillary differentiated thyroid carcinoma (papillary carcinoma group) and follicular differentiated thyroid carcinoma (follicular carcinoma group) to investigate the differences in expression of HBME-1. We further analyzed the correlation of the expression of HBME-1 in the papillary carcinoma group and the follicular carcinoma group with ultrasonic manifestation of thyroid. In both the papillary carcinoma group and the follicular carcinoma group, the levels of HBME-1 in affected tissues and the IHS score of HBME-1 expression were higher than those in the control group $(\mathrm{p}<0.05)$. In the papillary carcinoma group, the mean IHS score of HBME-1 expression in affected tissues was higher than in the follicular carcinoma group $(\mathrm{p}<0.05)$. There were no statistically significant differences in comparison to HBME-1 expression in affected tissues between the papillary carcinoma group and the follicular carcinoma group ( $\mathrm{p}>0.05)$. Between the papillary carcinoma group and the follicular carcinoma group, the differences in the comparison of the nodule diameter, echo, shape, boundary, calcification and blood flow signal were statistically significant $(\mathrm{p}<0.05)$, but incidence rate of enlargement of cervical lymph nodules between the groups were not statistically significant $(\mathrm{p}>0.05)$. Among patients in the papillary carcinoma group, the comparison of the nodule diameter, echo, shape, boundary, calcification and blood flow signal between
\end{abstract}

Correspondence to: Dr Shan Gao, Longnan Hospital of Daqing City, 35 Aiguo Road, Daqing, Heilongjiang 163453, P.R. China E-mail: gaoshan1188@163.com

*Contributed equally

Key words: differentiated thyroid carcinoma, human bone marrow endothelial cell marker-1, ultrasonic examination the HBME-1-positive patients and the HBME-1-negative patients showed no statistical significance ( $>0.05)$, but in the nodules of HBME-1-positive patients, the proportion of blood flow signal was higher than that in the nodules of HBME-1negative patients. Among patients in the follicular carcinoma group, there was no statistically significant differences in the comparison of ultrasonic manifestation of thyroid $(\mathrm{p}>0.05)$. Therefore, there are difference in HBME-1 expression and ultrasonic manifestations of thyroid in patients with papillary carcinoma and follicular differentiated thyroid carcinoma.

\section{Introduction}

Differentiated thyroid carcinoma is a common malignant tumor of the head and neck, of which papillary carcinoma and follicular carcinoma are the main pathologic types (1). In clinical practice, differentiated thyroid carcinoma is always characterized by relative high malignancy and a slow growth of tumor tissues. Surgical resection is the major procedure in the treatment of differentiated thyroid carcinoma, however, the invasion and metastasis of tumor cells are considered as major contributing factors to the recurrence of tumor and death of patients (2-4).

An early diagnosis of differentiated thyroid carcinoma is conducive to the prognosis of patients. Color Doppler Ultrasonography is a common non-invasive detection method which can help with early detection of thyroid node and thyroid carcinoma in clinical practice (5). Human bone marrow endothelium marker-1 (HBME-1), a common molecular marker of tumors, has been suggested for its potential use in diagnosis and prognosing differentiated thyroid carcinoma (6). Currently, there are few studies that report the expression levels of HBME-1 protein in different types of differentiated thyroid carcinoma tissues, and their correlation with ultrasonic manifestation of thyroid. Thereupon, we conducted immunohistochemistry (IHC) staining to detect the expression of HBME-1 in nodular goiter, differentiated papillary carcinoma and differentiated follicular carcinoma tissues, and compared the differences in HBME-1 expression among the three groups to investigate the correlation of HBME-1 expression in papillary carcinoma and follicular carcinoma with ultrasonic manifestations of thyroid. 


\section{Materials and methods}

Sample selection. We selected a total of 130 patients with thyroid diseases who were administered surgical resection at Longnan Hospital of Daqing City between April, 2014 and April, 2016. The subjects consisted of 50 patients with nodular goiter as the control group, 58 patients with papillary thyroid carcinoma as the papillary carcinoma group and 22 patients with follicular thyroid carcinoma as the follicular carcinoma group. The control group is composed of 22 males and 28 females, aged from 25 to 64 years with an average age of $46.7 \pm 2.2$ years. The papillary carcinoma group is composed of 22 males and 36 females, aged from 25 to 69 years with an average age of $46.9 \pm 2.6$ years. The follicular carcinoma group is composed of 10 males and 12 females, aged between 24 and 68 years with an average of $46.4 \pm 2.3$ years. The study was approved by the Ethics Committee of Longnan Hospital of Daqing City and informed consents were signed by the patients and/or guardians.

Ultrasound examination. In this study, 130 patients received the Color Doppler Ultrasonography (HI VISION Preirus; Hitachi, Tokyo, Japan) in which the frequency of the probe was set to $10-13 \mathrm{MHz}$. During the examination, in order to fully expose the thyroid, patients were required to stay in a supine position in order to observe the size, shape, boundary, calcification, blood flow and cervical lymph nodes of the nodules.

IHC. The expression of HBME-1 in the nodular goiter, differentiated papillary thyroid carcinoma and differentiated follicular thyroid carcinoma were detected using IHC. In this study, the rabbit anti-human HBME-1 polyclonal antibodies were used (1:500; cat. no. ab101139; Abcam, Cambridge, UK) The procedure was conducted in strict accordance with the instructions of rabbit anti-human HBME-1 polyclonal antibody kit.

Criteria (7) for IHC assessment were set as follows: Judgment was made according to the staining strength and area of positive cells, i.e.: i) 0 point for no color, ii) 1 point for shallow yellow, iii) 2 points for yellow, and iv) 3 points for medium brown. In each section, we randomly selected 5 typical regions as the vision field for cell count under the microscope (x400), and the results were averaged. The proportion of positive cells was used for scoring according to the following criteria: $0,0 \% ; 1,1-25 \% ; 2,26-50 \% ; 3,51-75 \%$; $4,76-100 \%$. The product of these two scores represented the IHC score, and the specimen with the IHC score $>6$ points was considered as positive.

Statistical analysis. The SPSS 20.0 (IBM, Armonk, NY, USA) software was used for statistical analysis. Measurement data are presented as mean $\pm \mathrm{SD}$. The Chi-square test was performed for comparison of measurement data, paired samples t-test was conducted for comparison of count data, and F-test for comparisons among three or more groups. $p<0.05$ suggested that the difference was statistically significant.

\section{Results}

Expression of HBME-1 in differentiated thyroid carcinoma tissues. Cells with HBME-1 expression had pale brown

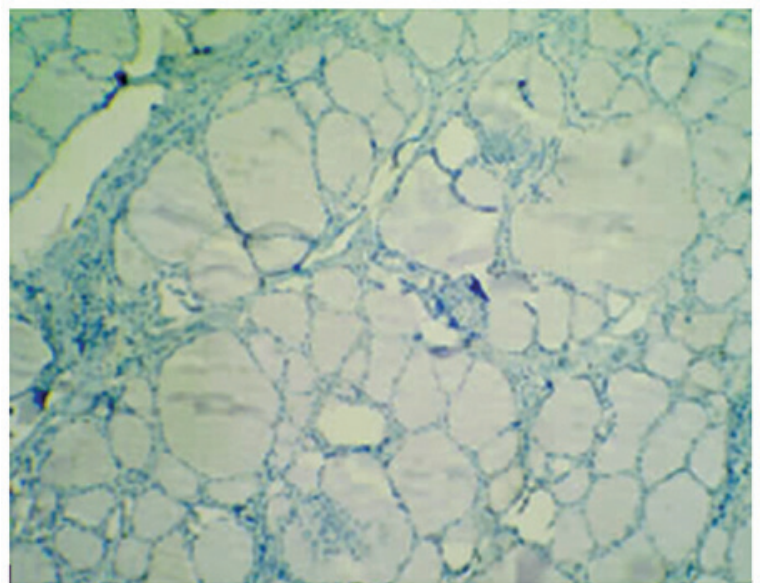

Figure 1. Human bone marrow endothelial cell marker-1 (HBME-1) negative in nodular goiter (SP, x400).

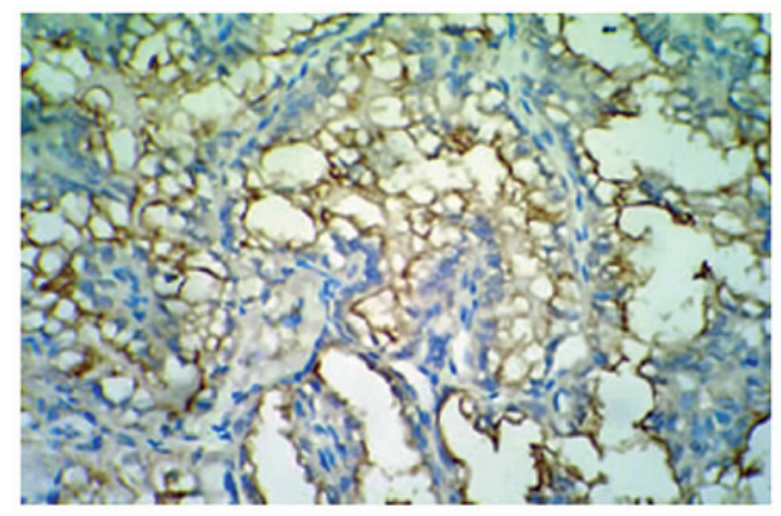

Figure 2. Human bone marrow endothelial cell marker-1 (HBME-1) positive in papillary thyroid carcinoma $(\mathrm{SP}, \mathrm{x} 400)$.

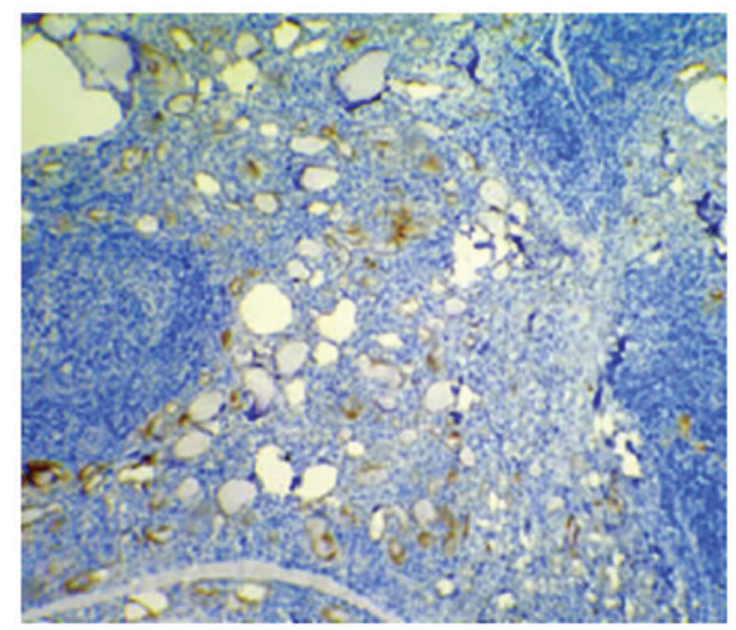

Figure 3. Human bone marrow endothelial cell marker-1 (HBME-1) positive in follicular thyroid carcinoma (SP, x400).

particles (Figs. 1-3). The level of HBME-1 expression and the average IHC score of HBME-1 expression in the papillary carcinoma group and the follicular carcinoma group were higher than those in the nodular goiter group $(p<0.05)$. In the papillary carcinoma group, the average IHC score in affected 
Table I. Comparison of HBME-1 expressions among three groups.

\begin{tabular}{|c|c|c|c|c|}
\hline \multirow[b]{2}{*}{ Group } & \multirow[b]{2}{*}{$\mathrm{n}$} & \multicolumn{2}{|c|}{ HBME-1 expression, $\mathrm{n}(\%)$} & \multirow{2}{*}{$\begin{array}{c}\text { Average HIS score of HBME-1 } \\
\text { expression (point) }\end{array}$} \\
\hline & & Positive & Negative & \\
\hline Control group & 50 & $13(26)$ & $37(74)$ & $3.12 \pm 1.65$ \\
\hline Papillary carcinoma group & 58 & $40(69)$ & $18(31)$ & $8.01 \pm 2.74$ \\
\hline Follicular carcinoma group & 22 & $15(68.2)$ & $7(31.8)$ & $5.56 \pm 2.18$ \\
\hline F-value & & 15.562 & 13.815 & 17.447 \\
\hline P-value & & $<0.05$ & $<0.05$ & $<0.05$ \\
\hline
\end{tabular}

HBME-1, human bone marrow endothelial cell marker-1.
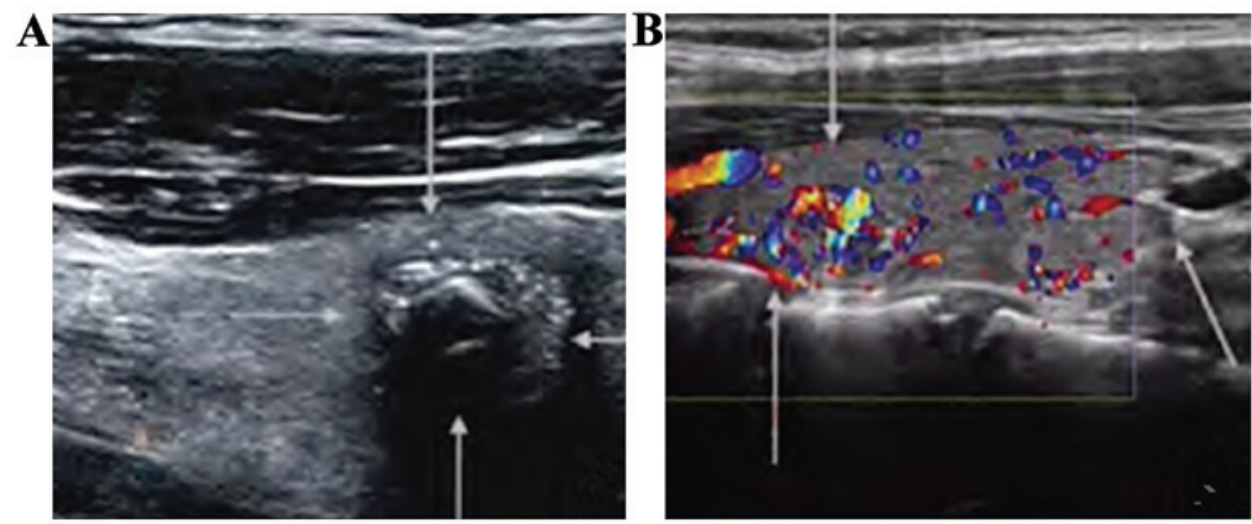

Figure 4. Ultrasonic manifestation of differentiated thyroid carcinoma (arrows for the range of mass): (A) Low echo, irregular shape, gross calcification and micro-calcification inside the nodules; (B) Abundant blood flow inside the nodules.

tissues was higher than that in the follicular carcinoma group $(\mathrm{p}<0.05)$. There were no statistically significant differences in comparison of the positive rate of HBME-1 expression between the papillary carcinoma group and the follicular carcinoma group $(\mathrm{p}>0.05)$ (Table I).

Comparison of ultrasonic manifestations of thyroid between the papillary carcinoma group and the follicular carcinoma group. When comparing the diameter, echo, shape, boundary, calcification and blood flow signal between the papillary carcinoma group and the follicular carcinoma group, differences were statistically significant $(p<0.05)$. However, the incidence of enlargement of cervical lymph nodules was not statistically significantly different between the two groups ( $>0.05$ ) (Table II and Fig. 4).

Correlation of HBME-1 expression in affected tissues with the ultrasonic manifestation of thyroid in the papillary carcinoma group and the follicular carcinoma group. Among patients in the papillary carcinoma group, we discovered that there were no statistically significant differences in the comparison of the diameter, echo, shape, boundary, calcification and enlargement of lymph nodules between the HBME-1 positive patients and HBME-1 negative patients $(\mathrm{p}>0.05)$. The proportion of HBME-1 positive patients with a signal of blood flow was higher than that in the HBME-1 negative patients (Table III). In patients of the follicular carcinoma group, the comparison of ultrasonic manifestations of thyroid between the HBME-1 positive patients and HBME-1 negative patients presented no statistically significant differences (p>0.05) (Table IV).

\section{Discussion}

In clinical practice, we always encounter patients that suffer from the thyroid lesions with atypical symptoms, such as papillary carcinoma or papillary hyperplasia, which possess some difficulties in differentiation of these atypical symptoms. For patients with thyroid micro-carcinoma that have a lesion with a diameter $<0.5 \mathrm{~cm}$, if there are not any characteristic papillary structures, then the collagen tissues would be distributed in the follicular structures under the microscope, and, accordingly, a differential diagnosis would be given to exclude the possibility of simple hyperplasia of collagen tissue (8-11). Clinical practice (12-14) has proven that IHC can be used for a differential diagnosis of papillary thyroid carcinoma. In recent years, more and more attention has been paid to some tumor markers with high sensitivity, such as cytokeratin 19 (CK19) and HBME-1. These markers have been applied in the clinical diagnosis of many malignant tumors, including thyroid carcinoma $(13,15,16)$.

HBME-1 is an antigen constituent of the microvilli on the surface of mesothelial cells in humans, and hyaluronic acid (HA) is the main ingredient of HBME-1 $(17)$. Studies $(18,19)$ have reported that the high expression of HBME-1 can be detected in papillary thyroid carcinoma tissues. In this study, 

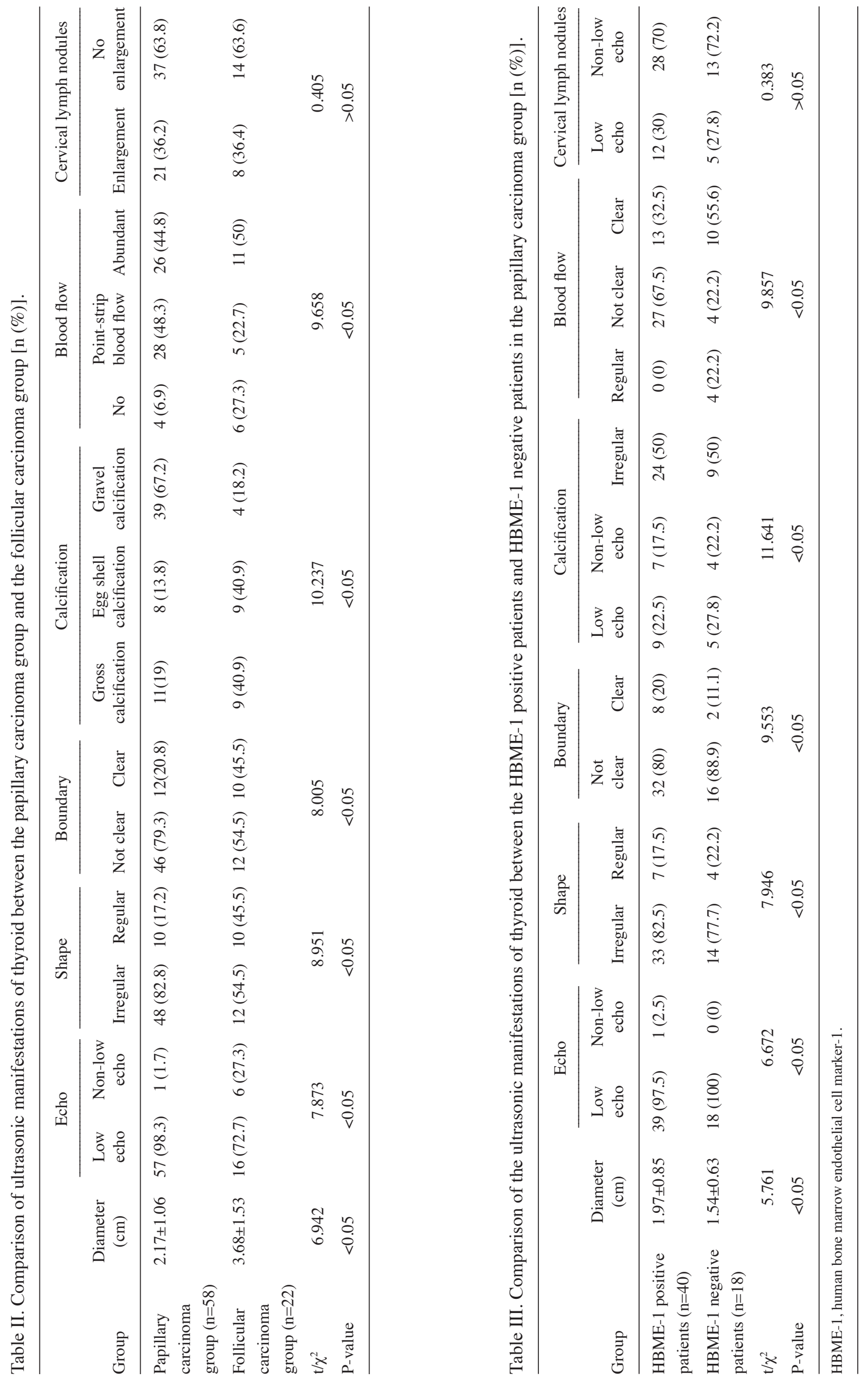
our results show that in the papillary carcinoma group and the follicular carcinoma group, the level of expression of HBME-1 in affected tissues and the IHC score of HBME-1 expression were all higher than those in the control group $(\mathrm{p}<0.05)$. In the papillary carcinoma group, the mean IHC score of HBME-1 expression in affected tissues was higher than that in the follicular carcinoma group $(\mathrm{p}<0.05)$. There were no statistically significant differences in comparison to HBME-1 expression in affected tissues between the papillary carcinoma group and the follicular carcinoma group $(\mathrm{p}>0.05)$. These results suggested that HBME-1 is highly expressed in these pathologic types (papillary and follicular) of thyroid carcinoma, and there may be no significant differences in the comparison of the positive rate of expression between different pathologic types of thyroid carcinoma. In addition, the IHC score of HBME-1 expression in papillary thyroid carcinoma tissues is significantly higher than that in the follicular thyroid carcinoma tissues, and therefore, we can infer that HBME-1 expression in papillary carcinoma tissues is remarkably higher than that in the follicular carcinoma tissues.

In recent years, color Doppler ultrasonography has been widely applied in clinical practice, which effectively improves the clinical diagnosis of malignant tumors in the thyroid. In clinical practice, the ultrasonic diagnosis of thyroid for judgment of the type of thyroid nodule (malignant or benign) is made through evaluating the shape and boundary of nodule, echo intensity, calcification and blood flow signals. Generally, ultrasonic manifestations of thyroid, including low echo, irregular shape, obscure boundary and micro-calcification, are considered as potential signs of thyroid carcinoma. In this study, our results show that between the papillary carcinoma group and the follicular carcinoma group, differences in the comparison of the nodule diameter, echo, shape, boundary, calcification and blood flow signal are statistically significant $(\mathrm{p}<0.05)$, however, there are no statistically significant differences in the comparison of incidence rate of the enlargement of cervical lymph nodules $(\mathrm{p}>0.05)$. Among patients in papillary carcinoma group, the difference in the nodule diameter, echo, shape, boundary, calcification and blood flow signal between the HBME-1-positive patients and the HBME-1-negative patients were no statistically significant $(\mathrm{p}>0.05)$. On the other hand, in the nodules of HBME-1-positive patients, the proportion of blood flow signal was higher than that in the nodules of HBME-1-negative patients. Among patients in the follicular carcinoma group, there was no statistically significant difference in the comparison of ultrasonic manifestation of the thyroid between the HBME-1 positive patients and the HBME-1 negative patients ( $>0.05)$. Our results indicate that there is increased blood flow in HBME-1 positive patients with papillary carcinoma, and there are no statistically significant differences in the comparison of ultrasonic manifestations of thyroid between the HBME-1-positive and -negative patients with follicular carcinoma.

In conclusion, in the tissues of papillary carcinoma and follicular differentiated thyroid carcinoma, there are differences in comparison of IHC scores of HBME-1 expression and some ultrasonic manifestation of thyroid. Among the papillary thyroid carcinoma patients, the blood flow signal of the HBME-1 positive patients is much higher than that of the HBME-1 negative patients. 


\section{References}

1. Asa SL, Giordano TJ and LiVolsi VA: Implications of the TCGA genomic characterization of papillary thyroid carcinoma for thyroid pathology: Does follicular variant papillary thyroid carcinoma exist? Thyroid 25: 1-2, 2015.

2. Mebed AH: Aggressive surgical therapy for locally invasive differentiated thyroid carcinoma: An experience of nineteen (19) cases. J Egypt Natl Canc Inst 19: 282-291, 2007.

3. Paschke R, Lincke T, Müller SP, Kreissl MC, Dralle H and Fassnacht M: The Treatment of Well-Differentiated Thyroid Carcinoma. Dtsch Arztebl Int 112: 452-458, 2015.

4. Xu D, Wang L, Long B, Ye X, Ge M, Wang K, Guo L and Li L: Radiofrequency ablation for postsurgical thyroid removal of differentiated thyroid carcinoma. Am J Transl Res 8: 1876-1885, 2016.

5. Cantisani V, Maceroni P, D'Andrea V, Patrizi G, Di Segni M, De Vito C, Grazhdani H, Isidori AM, Giannetta E, Redler A, et al: Strain ratio ultrasound elastography increases the accuracy of colour-Doppler ultrasound in the evaluation of Thy- 3 nodules. A bi-centre university experience. Eur Radiol 26: 1441-1449, 2016.

6. de Matos PS, Ferreira AP, de Oliveira Facuri F, Assumpção LV, Metze K and Ward LS: Usefulness of HBME-1, cytokeratin 19 and galectin-3 immunostaining in the diagnosis of thyroid malignancy. Histopathology 47: 391-401, 2005.

7. Sadamori H, Yagi T, Iwagaki H, Matsuda H, Shinoura S, Umeda Y, Ohara N, Yanai H, Ogino T and Tanaka N: Immunohistochemical staining of liver grafts with a monoclonal antibody against HCV-Envelope 2 for recurrent hepatitis $\mathrm{C}$ after living donor liver transplantation. J Gastroenterol Hepatol 24: 574-580, 2009.

8. Verslype C, Nevens F, Sinelli N, Clarysse C, Pirenne J, Depla E, Maertens G, van Pelt J, Desmet V, Fevery J, et al: Hepatic immunohistochemical staining with a monoclonal antibody against HCV-E2 to evaluate antiviral therapy and reinfection of liver grafts in hepatitis C viral infection. J Hepatol 38: 208-214, 2003.

9. Casey MB, Lohse CM and Lloyd RV: Distinction between papillary thyroid hyperplasia and papillary thyroid carcinoma by immunohistochemical staining for cytokeratin 19, galectin-3, and HBME-1. Endocr Pathol 14: 55-60, 2003.
10. Lee YS, Yun JS, Jeong JJ, Nam KH, Chung WY and Park CS: Thyroid hemiagenesis associated with thyroid adenomatous hyperplasia and papillary thyroid carcinoma. Thyroid 18: 381-382, 2008.

11. Dong S, Song XS, Chen G and Liu J: Mixed primary squamous cell carcinoma, follicular carcinoma, and micropapillary carcinoma of the thyroid gland: A case report. Auris Nasus Larynx 43: 455-459, 2016.

12. Bychkov A, Sampatanukul P, Shuangshoti S and Keelawat S: TROP-2 immunohistochemistry: A highly accurate method in the differential diagnosis of papillary thyroid carcinoma. Pathology 48: 425-433, 2016.

13. Erdogan-Durmus S, Ozcan D, Yarikkaya E, Kurt A and Arslan A: CD56, HBME-1 and cytokeratin 19 expressions in papillary thyroid carcinoma and nodular thyroid lesions. J Res Med Sci 21: 49-54, 2016.

14. Chen YJ, Zhao RM, Zhao Q, Li BY, Ma QY, Li X and Chen X: Diagnostic significance of elevated expression of HBME-1 in papillary thyroid carcinoma. Tumour Biol 37: 8715-8720, 2016.

15. Zhu X, Sun T, Lu H, Zhou X, Lu Y, Cai X and Zhu X: Diagnostic significance of CK19, RET, galectin-3 and HBME-1 expression for papillary thyroid carcinoma. J Clin Pathol 63: 786-789, 2010.

16. Liu Z, Yu P, Xiong Y, Zeng W, Li X, Maiaiti Y, Wang S, Song H, Shi L, Liu C, et al: Significance of CK19, TPO, and HBME-1 expression for diagnosis of papillary thyroid carcinoma. Int $\mathbf{J}$ Clin Exp Med 8: 4369-4374, 2015.

17. El-Mahdy MM, Mabrouk SH, El-Din ZS, Ghazal FA and Mohamed HH: Diagnostic value of HBME-1 and CK19 expression in papillary thyroid carcinoma, well-differentiated tumors of uncertain malignant potential, and benign thyroid nodules. Egypt J Pathol 31: 68-74, 2011.

18. Chao TT, Maa HC, Wang CY, Pei D, Liang YJ, Yang YF, Chou SJ and Chen YL: CIP2A is a poor prognostic factor and can be a diagnostic marker in papillary thyroid carcinoma. APMIS 124: 1031-1037, 2016

19. Yeşil C, Kandemir O, Haksever H and Dabakoğlu T: Is BECLIN-1 immunoreactivity more effective than HBME-1 in diagnosis of papillary thyroid cancer? Acta Chir Belg 115: 299-305, 2015. 